\title{
Detection of Resistance Phenotype and Gene of Avian Escherichia coli to $\beta$-lactam Antibiotics
}

\author{
Zhang Shilei, Bai Yun, Jia Ze, Wang Chunguang and Zhang Tie* \\ College of Animal Medicine, Agricultural University of Hebei, Baoding 071001, Hebei, China \\ *For correspondence: zhangtie1998@163.com
}

\begin{abstract}
Understanding and detecting $\beta$-lactam antibiotics resistance and resistance genes and elucidating the drug-resistant mechanism is of significance to prevention and treatment of avian Escherichia coli disease. In this study, the ESBLs (Extended Spectrum $\beta$-Lactamases) producing strains were screened from $41 \mathrm{E}$. coli isolates by double paper disk test; the $\beta$-lactam antibiotics resistance was investigated by Kirby-Bauer (K-B) drug susceptibility test; and three ESBLs genes including TEM, SHV and CTX-M were tested by Polymerase Chain Reaction. The results showed that there were 31 (75.6\%) ESBLs producing strains. The $E$. coli had highest resistance to penicillin and first generation cephalosporin followed by to second and third generation cephalosporins and monocyclic lactams. PCR revealed 73.2\% (30/41) of E. coli isolates carrying ESBLs gene. The positive rate of TEM, CTX-M and SHV were 63.4\% (26/41), 31.7\% (13/41) and 4.9\% (2/41), respectively. There were 10 strains $(24.4 \%)$ carrying more than two kinds of resistance genes. In conclusion, the drug resistance is directly related to the clinical use time and range of $\beta$-lactam antibiotics. The antibiotics resistance of $E$. coli isolated from avian to $\beta$-lactam in North China is mainly mediated by TEM and CTX-M genes. (C) 2017 Friends Science Publishers
\end{abstract}

Keywords: Avian Escherichia coli; $\beta$-lactam antibiotics; ESBLs gene; PCR

\section{Introduction}

In current animal husbandry, the prevalence of bacterial disease becomes more and more serious due to expansion of animal breeding scale, increase of raising density and stress factors. Escherichia coli is a major pathogen that can cause animal diarrhea and even lead to death of pigs and chickens. The antibiotics are often used for prevention and treatment of colibacillosis. The $\beta$-lactam antibiotic including penicillins, cephalosporins, cephamycins, monocyclic lactams and other atypical class refers to a large class of antibiotics containing the $\beta$-lactam ring structure. The $\beta$ lactam antibiotic is the most common antibacterial drug used by veterinarians. Due to the abuse of antibiotics and resistance gene transfer between bacteria, the drug resistance of bacteria is becoming more and more serious.

The main resistance mechanism of $E$. coli to $\beta$-lactam antibiotic is that it can produce ESBLs which can destroy the $\beta$-lactam ring through hydrolysis or non-hydrolysis and inactivate the antibiotic (George et al., 1991). The production of ESBLs enzyme is mainly mediated by the resistance genes located on chromosome and plasmid. As a result of the ESBLs gene and other resistance genes coexisted in one plasmid, the ESBLs gene is often involved in bacterial multi-drug resistance (De Champs et al., 2000). It has caused great difficulties to the clinical treatment of infectious diseases. At present, five types of ESBLs genes have been found including TEM, SHV, CTX-M, OXA and other type (Yuan et al., 2010). The bacteria producing ESBLs have different genotypes in different regions. In the United States and South Korea, the TEM and SHV are the main genotypes; SHV-2 and SHV-5 are mainly in Germany; CTX-M is mainly in Europe; and TEM, SHV and CTX-M are mainly in China (Ye et al., 2010). To monitor the $\beta$ lactam antibiotics resistance and to find out the mechanisms as well as to guide clinical medication, this study detected the $\beta$-lactam antibiotics resistance and ESBLs gene of avian E. coli isolated from North China.

\section{Materials and Methods}

\section{Reagents}

MHA medium and MacConkey medium, drug-sensitive paper discs were purchased from Hangzhou Tianhe Microorganism Reagent Co., Ltd (Hangzhou, China). PCR kits, Taq DNA polymerase, DNA Markers, dNTPs, agarose, Gold-view dye and other reagents were purchased from TaKaRa Biotechnology (Dalian) Co., Ltd. (Dalian, China).

\section{Isolation and Identification of $\boldsymbol{E}$. coli}

The livers, feces, pericardial fluid and other pathological materials of dead chickens were collected from North China 
under sterile conditions, including Beijing, Hebei and Shanxi regions. The collected specimens were diluted with sterile saline and then cross-inoculated in MacConkey medium. After culturing for 12 to $16 \mathrm{~h}$ at $37^{\circ} \mathrm{C}$, the single pink colony was picked and stained using Gram staining. After purification repeatedly, there were 41 isolates identified as avian $E$. coli by biochemical tests.

\section{Screening and Confirmatory Tests for ESBLs}

The screening of ESBLs producing strains was performed on the MHA medium by using disc diffusion method following Clinical and Laboratory Standards Institute (CLSI, 2011). Control strain E. coli ATCC25922 was supplied by China Institute of Veterinary Drugs Control (Beijing, China). The ceftazidime (CAZ), aztreonam (AZT), cefotaxime (CTX) and ceftriaxone (CRO) were used for initial screening test. The inhibition zone diameter of $\mathrm{CAZ} \leq$ $22 \mathrm{~mm}, \mathrm{AZT} \leq 27 \mathrm{~mm}, \mathrm{CTX} \leq 27 \mathrm{~mm}$ and $\mathrm{CRO} \leq 25 \mathrm{~mm}$ indicated the ESBLs production. The ESBLs phenotype was also confirmed by phenotypic confirmatory test. Two groups of drug-sensitive paper discs included (1) CAZ and CAZ plus clavulanic acid (CLA); (2) CTX and CTX plus CLA (single drug for $30 \mu \mathrm{g} /$ piece; compound drug for 30/10 $\mu \mathrm{g} /$ piece). An increase of $\geq 5 \mathrm{~mm}$ for inhibition zone diameter of either CAZ or CTX in the presence of CLA confirmed the ESBLs production.

\section{Drug Susceptibility Test}

The antibiotics investigated in the study included penicillins (ampicillin, carbenicillin and piperacillin), $1^{\text {st }}$ generation cephalosporins (cephalothin), $2^{\text {nd }}$ generation cephalosporins (cefuroxime, cefoxitin and cefaclor), $3^{\text {rd }}$ generation cephalosporins (ceftriaxone, cefotaxime and ceftazidime), $4^{\text {th }}$ generation cephalosporins (cefepime) carbapenems (imipenem) and monocyclic lactams (aztreonam). Drug susceptibility tests were done by using K-B method. The test liquid was coated on the MHA medium followed by placement of drug sensitive paper discs on the medium. After incubation for $24 \mathrm{~h}$ at $37^{\circ} \mathrm{C}$, the inhibition zone diameters were measured and drug resistance was calculated following CLSI (2011).

\section{PCR Amplification of $\beta$-lactam Antibiotic Resistance Genes}

The primers were designed (Table 1) according to Ye et al. (2010) and synthesized by Beijing Sunbiotech Co. Ltd., (Beijing, China). PCR was carried out using MyCycler thermal cycler (Bio-Rad, Hercules, CA) with boiling method to get the bacterial chromosomal DNA (Sambrook and Russell, 2008). PCR reaction was conducted in $50.0 \mu \mathrm{L}$ mixture containing 1.25 U Taq DNA polymerase, $0.8 \mathrm{mM} / \mathrm{L}$ dNTPs, $0.2 \mu \mathrm{M} / \mathrm{L}$ each primer, $2.5 \mu \mathrm{L}$ template DNA and $\mathrm{ddH}_{2} \mathrm{O}, 5 \mu \mathrm{L} 10 \times \mathrm{Taq}$ buffer was added to make the final volume as $50 \mu \mathrm{L}$. The PCR thermal cycles were as follows: initial denaturation temperature at $94^{\circ} \mathrm{C}$ for $5 \mathrm{~min} ; 32$ cycles of denaturation at $94^{\circ} \mathrm{C}$ for $50 \mathrm{~s}$, annealing at $55^{\circ} \mathrm{C}$ for $55 \mathrm{~s}$, and extension at $72^{\circ} \mathrm{C}$ for $60 \mathrm{~s}$; final extension temperature at $72^{\circ} \mathrm{C}$ for $5 \mathrm{~min}$. The amplification products were stored at $4^{\circ} \mathrm{C}$ until use.

\section{Gel Electrophoresis of PCR Products}

A $1.0 \mu \mathrm{L} 6 \times$ loading buffer were mixed with5.0 $\mu \mathrm{L}$ PCR products and subjected to electrophoresis on a $1.5 \%(\mathrm{wt} / \mathrm{vol})$ agarose gel at $80 \mathrm{~V}$ for $50 \mathrm{~min}$. The JY1000C universal electrophoresis power was purchased from Beijing Liuyi Instrument Factory (Beijing, China), GoLd-view was used as dye and DL-2000 DNA Marker was used as molecular weight standard. After electrophoresis, the gels were observed under WD-9413C UV gel imaging system (Beijing Sunbiotech Co. Ltd., Beijing, China). PCR products were sequenced and the sequences were analyzed by DNAStar (http://www.dnastar.com/).

\section{Results}

\section{Screening of ESBLs Producing Strains}

Results showed that the inhibition zone diameter of the control strain to each drug was in line with the CLSI standard. A total of 31 ESBLs producing strains were screened and confirmed from $41 \mathrm{E}$. coli isolates by screening and confirmatory tests with a detection rate of $75.6 \%(31 / 41)$.

\section{Avian $E$. coli Isolates Resistance to $\beta$-lactam Antibiotic}

Majority $(92.7 \%)$ of the E. coli isolates demonstrated resistance to penicillins and first generation cephalosporins. The sensitive and resistance rates to $2^{\text {nd }}$ and $3^{\text {rd }}$ generation cephalosporins and monocyclic lactams were close, but resistance rate to cefoxitin was very low. The $E$. coli isolates were extremely sensitive to carbapenems and $4^{\text {th }}$ generation cephalosporins. The sensitive rates to cefepime and imipenem were 87.8 and $100 \%$, respectively (Table 2).

\section{Distribution of $\beta$-lactam Antibiotic Resistance Genes in Avian E. coli Isolates}

DNA templates of $41 E$. coli isolates were prepared for ESBLs gene analysis. The ESBLs genes were amplified from $30 \mathrm{E}$. coli isolates (including carrying one and more than one resistance gene), and positive rate was $73.2 \%$ (30/41). Moreover, the TEM gene had the highest positive rate, up to $63.4 \%(26 / 41)$. The positive rate of CTX-M and SHV were $31.7 \%(13 / 41)$ and $4.9 \% \quad(2 / 41)$. The electrophoresis of PCR products from some isolates is shown in (Fig. 1-3). The amplified ESBLs genes had higher than $98 \%$ nucleotide sequence similarity to the 
Table 1: Characteristics of primer pairs specific for ESBLs genes

\begin{tabular}{lll}
\hline Resistance genes & Pimer sequence $\left(5^{\prime} \rightarrow 3^{\prime}\right)$ & Size $(\mathrm{bp})$ \\
\hline TEM & Forward: CAGAAACGCTGGTGAAAGTA & 719 \\
& Reverse: ACTCCCCGTCGTGTAGATAA & \\
CTX-M & Forward: AGTGAAAGCGAACCGAATC & 365 \\
& Reverse: CTGTCACCAATGCTTACC & \\
SHV & Forward: ATGCGTATATTCGCCTGTG & 502 \\
& Reverse: CCTCATTCAGTTCCGTTTCC & \\
\hline
\end{tabular}

Table 2: Resistance rate of avian $E$. coli isolates to $\beta$ lactam antibiotics

\begin{tabular}{|c|c|c|c|}
\hline \multirow{2}{*}{ Antibiotics } & \multicolumn{3}{|c|}{ Resistance rate $(\%)^{\mathrm{a}}$} \\
\hline & Resistant & Intermediate & Sensitive \\
\hline Ampicillin & $92.7(38 / 41)$ & $7.3(3 / 41)$ & $0(0 / 41)$ \\
\hline Carbenicillin & $90.2(37 / 41)$ & $4.9(2 / 41)$ & $4.9(2 / 41)$ \\
\hline Piperacillin & $78.1(32 / 41)$ & $12.2(5 / 41)$ & $9.8(4 / 41)$ \\
\hline Cephalotin & $75.6(31 / 41)$ & $12.2(5 / 41)$ & $12.2(5 / 41)$ \\
\hline Cefuroxime & $48.8(20 / 41)$ & $26.8(11 / 41)$ & $24.4(10 / 41)$ \\
\hline Cefoxitin & $9.8(4 / 41)$ & $17.1(7 / 41)$ & $73.2(30 / 41)$ \\
\hline Cefaclor & $48.8(20 / 41)$ & $12.2(5 / 41)$ & $39.0(16 / 41)$ \\
\hline Ceftriaxone & $43.9(18 / 41)$ & $19.5(8 / 41)$ & $36.6(15 / 41)$ \\
\hline Cefotaxime & $34.2(14 / 41)$ & $26.8(11 / 41)$ & $39.0(16 / 41)$ \\
\hline Ceftazidime & $34.2(14 / 41)$ & $24.4(10 / 41)$ & $41.5(17 / 41)$ \\
\hline Cefepime & $7.3(3 / 41)$ & $4.9(2 / 41)$ & $87.8(36 / 41)$ \\
\hline Imipenem & $0(0 / 41)$ & $0(0 / 41)$ & $100(41 / 41)$ \\
\hline Aztreonam & $41.5(17 / 41)$ & $22.0(9 / 41)$ & $36.6(15 / 41)$ \\
\hline
\end{tabular}

corresponding sequences available in the GenBank database [ID: AY956315 (TEM gene), AY293071 (CTX-M gene) and EF650037 (SHV gene)].

\section{Distribution of Multiple $\beta$-lactam Antibiotic Resistance Genes in Avian E. coli Isolates}

Of 41 E. coli isolates, $48.8 \%$ (20/41) carried a single resistance gene; $22.0 \%(9 / 41)$ had two resistance genes and $2.4 \%(1 / 41)$ had three resistance genes. In addition, a total of 11 isolates accounting for $26.8 \%$ (11/41) had no target gene (Table 3).

\section{Discussion}

This study isolated 41 avian $E$. coli strains and detected their resistance to $\beta$-lactam antibiotics. The results showed that the resistance to penicillins and first generation cephalosporins was serious. The amount of resistance strains to $2^{\text {nd }}$ and $3^{\text {rd }}$ generation cephalosporins and monocyclic lactams was close to that of sensitive strains. The most of isolated strains were extremely sensitive to $4^{\text {th }}$ generation cephalosporins and carbapenems. These results indicate that the avian $E$. coli had different drug resistance to different types of $\beta$-lactam antibiotics. Therefore, this drugresistance regularity is directly related to the clinical use time and range of $\beta$-lactam antibiotics. The longer use time, the larger use range, and the more serious resistance.
Table 3: Detection of $\beta$-lactam resistance genes

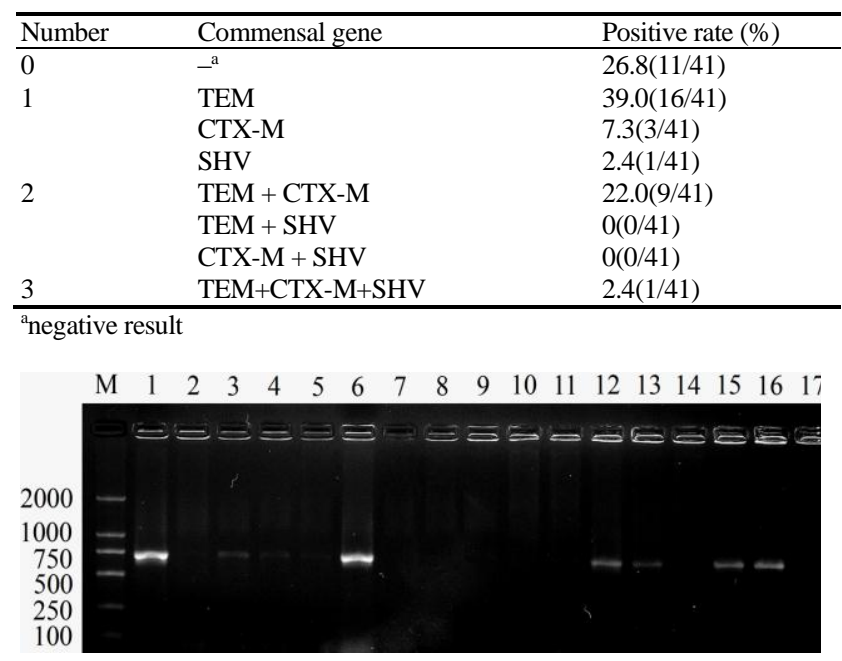

Fig. 1: PCR products of TEM gene

M: DNA Marke;Lanes 1-17:1-17 strains;Lanes 1,3-6,12,13,15,16 have the specific bands (719bp)

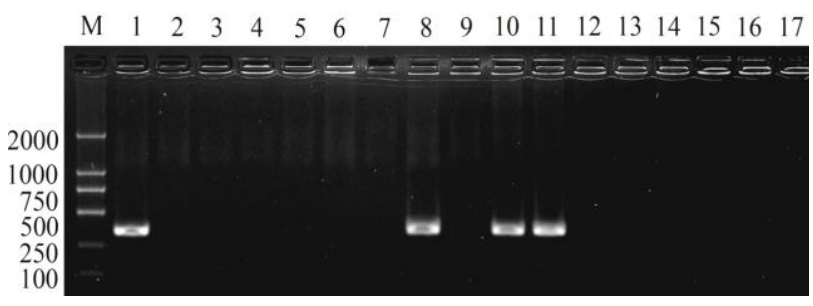

Fig. 2: PCR products of CTX-M gene

M: DNA Marke;Lanes 1-17:1-17 strains;Lanes 1,8,10,11 have the specific bands (365bp)

$\begin{array}{llllllllllllllllll}\mathrm{M} & 1 & 2 & 3 & 4 & 5 & 6 & 7 & 8 & 9 & 10 & 11 & 12 & 13 & 14 & 15 & 16 & 17\end{array}$

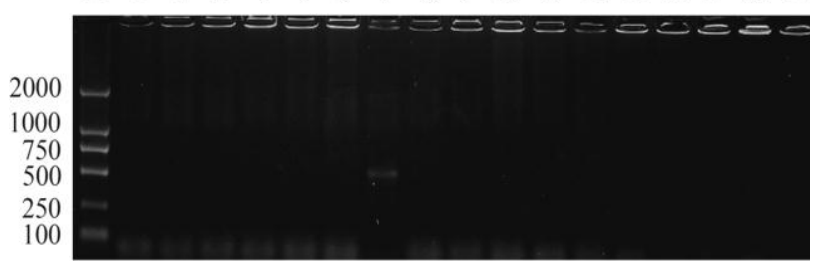

Fig. 3: PCR products of SHV gene

M: DNA Marke;Lanes 1-17:1-17 strains;Lanes 7 have the specific bands (502bp)

CLSI has reminded that the E. coli may be transformed from sensitive strain into resistance strain with the long time use of $3^{\text {rd }}$ generation cephalosporins, and this transformation can be produced within 3-4 d after treatment (CLSI, 2011). It was found that the ESBLs producing strains are not only resistant to $2^{\text {nd }}$ and $3^{\text {rd }}$ generation cephalosporins generally but also to fluoroquinolones, tetracyclines and aminoglycoside (Tian et al., 2011). Fu et al. (2007) have reported that ESBLs producing strains and Klebsiella pneumoniae are only sensitive to carbapenems including imipenem and meropenem. Moreover, the $\beta$-lactamase 
inhibitors such as clavulanic acid or tazobactam can reduce the minimal inhibitory concentration (MIC) of drugs.

The resistance genes were detected by PCR, and $73.2 \%(30 / 41)$ of $E$. coli isolates carried ESBLs gene and they were ESBLs producing strains. The TEM gene had the highest positive rate, up to $63.4 \%$ (26/41). The positive rate of CTX-M and SHV were $31.7 \%$ (13/41) and $4.9 \%(2 / 41)$, respectively. There were 10 strains carrying more than two kinds of resistance genes. It was verified that the $\beta$-lactam antibiotics resistance of avian E. coli in North China is mainly mediated by TEM and CTX-M genes. The previous study has reported that the TEM gene accounts for $53 \%$ in E. coli (Xiao et al., 2005). The TEM gene was amplified in ESBLs producing strains, but no SHV gene was found (Zhang et al., 2009). SHV gene is mainly carried by $K$. pneumoniae. However, it was amplified in avian E. coli, indicating that the resistance genes can transfer and exchange between different species of bacteria.

\section{Conclusion}

Studies on the drug-resistance and resistance gene of avian E. coli can guide clinical medication in North China and provide reference for research of $\beta$-lactam antibiotics resistance regularity of $E$. coli.

\section{Acknowledgements}

This study was financially supported by the National Natural Science Foundation of China (No. 31572560); the Hebei Key Technology R\&D Program grant (No. 16222701D) from Department of Science \& Technology, Hebei Province, China.

\section{References}

CLSI, 2011. Performance Standards for Antimicrobial Susceptibility Testing; Twenty-first Informational Supplement M100-S21, pp: 45-50. Clinical and Laboratory Standards Institute, Wayne, Philadelphia, USA

De Champs, C., D. Sirot, C. Chanal, R. Bonnet, J. Sirot and the French Study Group, 2000. A 1998 survey of extended-spectrum $\beta$ lactamases in Enterobacteriaceae in France. Antimicrob. Agents CH., 11: 3177-3179

Fu, X.L., H. Wu, H.Y. Chen, L. Yuan, Y.S. Pan, B.D. Wu and G.Z. Hu, 2007. Detection of extended-spectrum $\beta$-lactamases and AmpC enzyme and antibiotics susceptibility test analysis of pathogens isolated from poultry. J. Huazhong Agric. Univ., 4: 217-222

George, A., M.D. Jacoby, L. Gordon and M.D. Archer, 1991. New mechanisms of bacterial resistance to antimicrobial agents. N. Eng. J. Med., 324: 601-612

Sambrook, J. and D.W. Russell, 2008. The condensed protocols from molecular cloning, In: A Laboratory Manual, p: 197. Huang, P.T. (ed.). Chem. Ind. Press, Beijing, China

Tian, G.B., H.N. Wang, A.Y. Zhang and Y. Zhang, 2011. Detection of resistance to $\beta$-lactams and characterization of extended-spectrum lactamases in E. coli isolates from swine. Chin. J. Prev. Vet. Med., 10: 776-780

Xiao, Q.Z., D.H. Su, J.H. Jiang and N.S. Zhong, 2005. Study on the genotypes of TEM-like and SHV-like extended-spectrum betalactamases in clinical isolates of Gram-negative bacilli in Guangzhou. Chin. J. Lab. Med., 10: 1010-1014

Ye, M.Y., H.N. Wang, G.B. Tian, A.Y. Zhang, Y.W. Zhao and Y.S. Zhou, 2010. Detection of $\beta$-lactamases-resistant genes in Salmonella and $E$ coli isolated from swines and chickens. Chin. J. Vet. Med., 3: 15-17

Yuan, L., J.H. Liu, G.Z. Hu, Y.S. Pan, J. Mo, Y.J. Wei and Y.L. Pei, 2010. Genotypes of TEM-type and CTX-M-type extended-spectrum $\beta$ Lactamases produced by E. coli isolated from the fowl. Sci. Agric. Sin., 20: 4310-4316

Zhang, C.H., X.F. Zhang, X.G. Zhang, C.B. Zhao and J.H. Wang, 2009. Genotype detection of ESBLs in bacterium from swine and avian. Chin. Agric. Sci. Bull., 1: 8-11

(Received 22 December 2016; Accepted 03 January 2017) 